\title{
ÉTICA Y EMPRESA: LA CIUDADANÍA Y LOS FINES DE LA EMPRESA'
}

\author{
MARÍA SOLEDAD ESCALANTE BELTRÁN
}

- Universidad Antonio Ruiz de Montoya SJ, Lima-Perú

\section{RESUMEN}

La determinación del horizonte de finalidades y la inserción de la empresa en la realidad son momentos constitutivos de la existencia de la empresa que amplifican la mirada tradicional sobre la empresa. La mirada tradicional hace de la empresa una entidad ajena y extraña a la comunidad, la sociedad, la ciudadanía y el Estado. Conceptos como «cooperación»e «inclusión social» resultan chocantes para la mirada tradicional, presa como está todavía de la retórica capitalista que durante el siglo XX propagó, hasta naturalizar, las nociones de "competencia" y "libre mercado". La propagación y la naturalización fueron efectivas, pues logró su cometido, esto es difundir y asentar prejuicios que terminaron por reducir el alcance de la iniciativa empresarial a sólo una de sus múltiples dimensiones: el "afán de lucro". La empresa del siglo XXI tiene a su favor la experiencia de la incertidumbre que hace vacilar, y muchas veces retroceder, al capital financiero en contextos críticos; dispone, además, de los medios y los recursos para superar la obsolescencia del viejo modelo, que amenaza la sostenibilidad de la propia empresa porque la sitúa de espaldas a la realidad y desentendida del impacto real que tienen sus actividades en la vida de las personas, en la creencia de que, desvinculada (teóricamente) del Estado, vive ya la utopía del "mundo postpolítico y apolítico" donde se impone la hobbesiana "ley del más fuerte", que opta por la imposición autoritaria y la violencia cuando renuncia a la cooperación, al diálogo y la búsqueda consciente y consistente de puntos de confluencia que permitan establecer acuerdos que faciliten la convivencia pacífica.

Palabras clave: Misión de la empresa, Relación empresa sociedad, Ética empresarial

1. UPC Review of Global Management, Volumen 3, Número 1, junio 2017

\section{ABSTRACT}

The determination of the purpose horizon and the insertion of the company into reality, properly considered, amplifies the traditional view of the company, which made it strange and alien to concepts such as "cooperation" and "social inclusion", still prey to capitalist rhetoric that during the 20th century propagated the notions of "competition" and "free market" to spread prejudices that ended up reducing the scope of entrepreneurship to one of its many dimensions: the "profit motive". The company of the 21st century has in its favour the experience of the uncertainty that makes the financial capital in critical contexts waver, and often regress, and has the means and the resources to overcome the obsolescence of the model that threatens the sustainability of one's own because it places it back to reality and disregarded the real impact of its activities on people's lives, in the belief that, unrelated (theoretically) from the State, the utopia of the "post-political and apolitical world" already lives imposes the Hobbesian "law of the strongest", who opts for authoritarianism infliction and violence when he renounces cooperation, dialogue and a conscious and consistent search for points of convergence that allow the establishment of agreements that facilitate peaceful coexistence.

Key words: Purpose of the firm, Society - Firm relationship, Business ethics

En los hechos, la relación entre empresa, Estado, individuo y comunidad sigue siendo fructífera como al comienzo. Es necesario, por ello, repensar la finalidad de la empresa de cara a la conformación de una sociedad distinta. Ciertamente, resulta problemático que se haya naturalizado la noción de "competencia" como mediadora de las relaciones entre las personas, pues contribuye al debilitamiento de la ciudadanía, reduce el espacio público y limita la iniciativa privada a un horizonte estrecho que inhibe de un mayor despliegue y aprovechamiento de las virtualidades de la empresa. 
"Los ciudadanos modernos, ahora entregados a sus propósitos particulares, establecen como pauta de vida la competencia universal; por puestos de trabajo, por oportunidades de inversión, por productos de consumo o por lo que fuere. Mediados por la competencia, los individuos no perciben al otro como un conciudadano con quien comparten un horizonte de vida común; lo perciben precisamente como "otro", una potencial amenaza para lo propio o para lo que pudiera llegar a ser propio. La política no es por sí misma capaz de armonizar estas voluntades, no representa más un bien supremo concreto que tenga el poder para lograr que los individuos subordinen sus apetencias particulares a la pauta de la ciudadanía y a la perspectiva de lo común» (Del Águila, 2007, p. 220).

La empresa ha olvidado su vínculo con la realidad. Con ese olvido no solo corre el riesgo de desarraigarse, sino que pone en peligro su propia sostenibilidad en el medio y largo plazo. ¿Por qué es importante este problema? La siempre urgente y concomitante crisis de valores que atravesamos pone en evidencia la debilidad y la precariedad del viejo modelo empresarial y llama la atención sobre la importancia de re-anclar a la empresa en la realidad problematizando la cuestión de sus propósitos y finalidades en la hora actual.

Estudios previos señalan la importancia de la ética para la mejora del desempeño de la empresa frente al desafío que implica el reconocimiento responsable de su apertura hacia el individuo, el ciudadano, la comunidad y el Estado. Si bien todavía los avances y desarrollos teóricos son incipientes dada la reciente inserción de la categoría ética empresarial en la reflexión crítica contemporánea y que hace falta todavía una presentación sinóptica de su desarrollo, todo indica que existe la preocupación en las mismas empresas de incorporar la ética a sus procesos internos y externos. Este trabajo quiere aportar al esclarecimiento de esta línea de reflexión.

En ese sentido, ¿cuáles son las hipótesis y objetivos primarios y secundarios del presente estudio y cuáles son, si los hay, los vínculos con la teoría? A través de la noción aristotélica de télos, este trabajo se propone discernir la finalidad o el propósito de la empresa para garantizar su supervivencia de cara a la crisis que atraviesa (cf. Groppa, 2005; Nussbaum, 2002). La triple dimensión comunitaria cooperativa y socioeconómica de la empresa la delinea y configura frente a las versiones de la otredad que representan el individuo, la comunidad, el ciudadano, el Estado y las otras empresas. El artículo contrapone críticamente dos formas opuestas de comprender a la empresa moderna y contemporánea. El objetivo es reflexionar sobre la dimensión sociopolítica de la empresa, dimensión que es constitutiva e inalienable de su ser. Todavía la observación de esta dimensión se halla en un estadio incipiente en la investigación, pero no es imposible notar que la profundización y el discernimiento favorecen la explicitación del vínculo crítico entre la empresa, el entorno y sus actores.

¿Cómo se relacionan entre sí las hipótesis y el diseño de la investigación? En el Perú, la inserción de la empresa está regulada por el Estado soberano a través de los protocolos y normativas actuales y vigentes. Hay dos tendencias en cuanto a la reacción de la empresa frente a la presencia del Estado. Por una parte, la tendencia que rechaza todo vínculo con el Estado y promueve que este sea reducido a su mínima expresión, hasta que, eventualmente, se extinga y desaparezca. Por otra parte, la tendencia críticoconstructiva que reconoce la vitalidad del vínculo y la necesidad de su génesis histórica, así como la relevancia y la pertinencia de repensar, sobre una base real y realista, el propósito de la empresa.

En cuanto a las implicaciones teóricas y prácticas del presente estudio es preciso anotar que identifica el vínculo y la relación compleja entre la empresa y la eticidad en un contexto de crisis. Si bien la crítica ha desarrollado el sentido de la crisis, hasta ahora se ha concentrado más en los aspectos conflictivos que en los aspectos sinérgicos. El balance y contrapeso crítico de ambos aspectos contribuye al descernimiento de la crisis y puede contribuir a sentar las bases para superarla.

\section{INSERCIÓN DE LA EMPRESA EN LA REALIDAD}

El empresario crea la empresa y la inserta en la realidad a través de un proceso jurídico-normativo que genera inmediatamente consecuencias legales, morales, económicas, financieras y tributarias. Lo empresarial se asienta sobre la base de principios éticos que serán el soporte de la práctica reflexiva que necesita desarrollar el empresario para desenvolverse en la realidad económicosocial que es el contexto concreto donde realiza sus actividades empresariales. Es claro, naturalmente, que la empresa no está flotando a la deriva en el espacio imaginario del empresariado, sino que es una entidad real que da pie a consecuencias de distinto tipo en la realidad. No solo las consecuencias referidas a su actividad productiva intrínseca, hacia el interior de la empresa, sino también hacia el exterior, pues finalmente la empresa tiende hacia el otro en procura de satisfacerle una necesidad (Cortina, 1994)

Ética y empresa son en sí mismos conceptos complejos. Tanto más lo es, aun, su relación y su interacción. En los últimos treinta años ha habido, ciertamente, un incremento notable y sostenido de la bibliografía sobre la ética en el Perú, aunque todavía los avances de la investigación resultan incipientes y requieren mayor profundidad en su desarrollo (Sobrevilla 2009). La profundización avanza en el apuntalamiento de los aciertos explicativos de la crítica y en la corrección y enmienda del proceso en los experimentos fallidos. De ahí la necesidad de volver a las preguntas que subyacen a nuestros usos y hábitos con los términos «ética» y «empresa»: ¿qué es la ética?, ¿qué es la empresa?, ¿qué relación hay entre ellas?

Estas preguntas ofrecen los presupuestos para el establecimiento del diálogo y del debate que, finalmente, sirven al discernimiento de las cuestiones más problemáticas que aparecen como desafíos para la investigación (Del Mastro 2014). Debilitados por el uso y la escasa o nula reflexión, nuestras nociones y conceptos se desgastan y deslucen; poco a poco su sentido palidece y su significado resulta desleído. Quizá no haga falta una interpretación restrictiva de la noción de ética para pensar, en su seno, la empresa en general y su modo de ser en el 
mundo actual. La ética permite que la empresa comprenda dimensiones de la realidad que hasta ahora se sustraían a su mirada por la falta de métodos de observación adecuados.

\section{PROPÓSITO DE LA EMPRESA}

Los fines con lo que fue creada la empresa definen sus actividades, que conservan siempre el ser constitutivo de la empresa, y le imprimen sentido y dirección al rumbo de acción de la empresa, de modo que esta siga siendo la misma y fortalezca, en tiempo, su identidad. Finalmente, aunque la diversidad de empresas se rija por el mismo marco normativo, no hay dos empresas iguales e idénticas.

Si bien la empresa capitalista busca la generación de las utilidades y su constante incremento (cf. Calvo, 2014, p. 11), no representa una excepción al principio constitutivo de la empresa no solo como expresión de una voluntad común cooperativa (cf. Habermas, 1999, pp. 61-62), sino también de la empresa misma como producto de la cooperación. En efecto, la empresa capitalista nace a la sociedad moderna a través del régimen que determina el Estado soberano a través de sus normas para el establecimiento de actividades, impuestos, alcabalas y tributos (cf. Knieper, 1991; Habermas, 1999, p. 157)

En ese sentido, observamos, con Hegel (1993, p. 619, § 183), que empresa, Estado y sociedad, además de escuela, familia y vecindad, interactúan en el espacio de la moralidad y de la eticidad para formar y modelar a los miembros de la comunidad de acuerdo a principios que valoran positivamente la convivencia y la coexistencia pacífica de sus integrantes como fortalezas para apuntalar la cosa pública, lo común (cf. Habermas, 1999, pp. 61-62), como aquello que es de interés de toda la comunidad, no solo en abstracto, sino también en concreto: se trata de una comunidad consciente de su propia tradición y de su propio proceso constructivo, histórico (cf. Taylor, 1993, pp. 87,88-89; citado por Habermas, 1999, p. 193).

Ahora bien, si, por una parte, se destaca el carácter formal de la creación e inserción de la empresa como entidad abstracta, jurídica, existente en los registros que dispone el Estado para que la empresa asiente su existencia, por otra parte no se olvida que la abstracción de la empresa se hace tangible en las personas que trabajan en la empresa, para la fempresa y con la empresa, aun cuando cada individuo trabajara, no obstante, para sus propios fines específicos, pues cada individuo está urgido por satisfacer sus necesidades básicas personales, y no solamente las básicas.

Si bien es cierto que la fundación de la empresa decide de alguna manera su identidad en lo sucesivo, no es sino hasta que la empresa se ha asentado en el mercado y se ha consolidado que los rasgos de su identidad y de su "personalidad" resultan acentuados según el carácter y el temple de sus fundadores, gestores y directores. Recordar que la empresa moderna opera siempre bajo un enfoque constructivista (cf. Rendtorff, 2013) permite observar que, en su desarrollo y trayectoria, mientras gana la experiencia necesaria y la integra críticamente a sus procesos para lograr un mejor desempeño y mayor eficiencia, la empresa se reconoce como distinta de otras empresas y apuntala todo aquello que le permite distinguirse de otras empresas.
Cada empresa se define en parte por negación y diferenciación - no es aquella, sino esta empresa - en parte por afirmación y superación de los obstáculos que presenta la realidad, como pueden serlo la concurrencia de varias empresas ante la demanda de un consumidor o la presencia de muy escasos consumidores ante la oferta de las empresas. Si bien la relación entre empresa y cliente (o consumidor) puede no ser recíproca ni simétrica, esta relación no se reduce solamente al intercambio de dinero por mercadería (Calvo, 2014, p. 11; Cortina, 1994, en Del Águila, 2007, p. 254). No, por lo menos, desde el lado del cliente, que asocia un producto de una marca o de una empresa a una experiencia sensorial y lo representa en una serie de hábitos en el consumo y en el imaginario que ha construido en torno a los productos y servicios de determinadas marcas. Las empresas ponen atención al carácter "ritual" del consumo y disponen de las herramientas para canalizarlo a favor del incremento de sus utilidades a través de la publicidad personalizada (cf. Abalde, 2016; Beauchamp, 2000, pp. 476-485).

Para Cortina (1994), lo empresarial y lo cívico se implican mutuamente. El fortalecimiento de las instituciones cívicas de la democracia liberal ha favorecido el despliegue y crecimiento de la actividad empresarial y, por consiguiente, no solo el incremento de las empresas, sino también la mejora y el perfeccionamiento gradual del saber hacer empresa, que se consolida no solo como saber acumulado sino como tradición nutricia que favorece que la empresa afronte con integridad los retos que avizora de cara al futuro. La palabra "fin", que traduce la noción aristotélica télos, tiene, por un lado, la acepción de término, límite del acabamiento, punto máximo de perfeccionamiento (es decir areté, excelencia o virtud de una cosa); por otro lado, la palabra "fin" también tiene el sentido de finalidad, propósito, meta. Según Aristóteles, "la ética es una especie de la política» (Ética Nicomáquea, 1094b10). Es, pues, evidente, en este estadio de la reflexión, que no podemos pensar a la empresa ni completamente desconectada ni completamente aislada de la comunidad.

\section{¿MUNDO POSTPOLÍTICO Y APOLÍTICO? EMPRESA Y COMUNIDAD: COOPERACIÓN E INCLUSIÓN SOCIAL}

La empresa crea una comunidad de consumidores alrededor de sus productos (cf. Bourdieu, 1985, pp. 723744). Pero la empresa no solo tiene un vínculo con los consumidores de sus productos, sino que también se vincula con los intermediarios que facilitan sus procesos productivos para que la empresa consiga su objetivo de satisfacer la demanda de los consumidores. Frente a la imagen de la empresa omnipotente y autosuficiente - una imagen, en verdad, que poco se ajusta a la realidad-. la empresa aparece en el polo de la demanda y de la imposible autosuficiencia porque ella también necesita satisfacer necesidades que garanticen su subsistencia y su permanencia en el mercado.

El éxito dependerá de la capacidad de la empresa para adaptarse y consolidarse en la comunidad de ofertantes y productores que le brindan los insumos y los servicios que ella, la empresa, por sí misma no puede producir, bien porque no ha desarrollado un proceso, bien porque 
los insumos y los servicios estén lejos de su específica capacidad productiva.

El éxito de la empresa, en el paradigma de inspiración hobbesiana, consiste en superar a la "competencia universal" (Del Águila, 2007, p. 220) a toda costa para estar en el primer lugar en el mercado y liderar la tendencia siempre ascendente que señala el camino hacia la bonanza y la riqueza infinita (cf. Habermas, 1999, pp. 102-103). La diversidad de iniciativas empresariales y la capacidad de los empresarios de establecer alianzas estratégicas de desarrollo del mercado y profundización del sector productivo desmienten al viejo paradigma. Además, el Estado siempre es un activo promotor de la iniciativa empresarial a través de los incentivos tributarios y financieros (Arellano, 2011, 2013; Brescia \& Quea, 2014; De Echave \&Torres, 2005; Pastor et al., 2012).

Ante la caída del ídolo de la empresa absoluta, basada en el afán de lucro como único principio, la diversidad de las iniciativas empresariales se extiende en toda su amplitud mostrando que la empresa se puede constituir sobre principios distintos y de contenido menos conflictivo y contradictorio: la especialización del proceso productivo va de la mano con la especialización de la empresa a partir de las necesidades reales y concretas del consumidor.

Claro que una empresa puede crear nuevas empresas y albergarlas dentro de algún tipo de organización corporativa. Incluso puede descubrir la necesidad que se le sustrae al propio y todavía potencial consumidor, inconsciente de su necesidad, que solo el empresario pone al descubierto cuando opera sus patentes. Sin embargo, la empresa titánica y todopoderosa del "modelo" tendría que ser capaz de satisfacer plenamente las necesidades y tendría que ser la única capaz de hacerlo.

No se constata en la realidad una empresa tal. Dejando de lado los modelos fallidos y por eso mismo perfectibles, el viejo modelo ocultaba el modelo de la empresa ideal que queda como un paradigma que expresa la búsqueda de la excelencia empresarial que mueve a los empresarios más allá del afán de lucro (cf. Calvo, 2014, p. 11; Cortina, 1994, en Del Águila, 2007, p. 254; Scalzo, 2017, p. 32) y los sitúa en otro plano de reflexión acerca de la comprensión crítica de la empresa. Se trata de una ventaja comparativa que brinda una mejor visión en el mercado. Cabe anotar, además, que el conocimiento que producen las empresas no siempre es valorado oportunamente.

\section{HACIA UNA SOCIEDAD DISTINTA}

Frente al empresario ideal, una figura vacía como un cuenco, y que no se entera de nada, el empresario real sabe que, en última instancia, el ritmo productivo de la empresa depende del ritmo vital, la disposición natural del ánimo y el carácter de sus trabajadores. De ahí la importancia de que la empresa en los últimos años haya tomado conciencia cada vez más clara de la necesidad de estudios que les informen y les brinden insumos para reflexionar sobre cómo mejorar el clima laboral, el trato de la empresa hacia los trabajadores y sus familias, sobre cuyo estilo de vida impacta directamente independientemente de la precariedad o seguridad laboral en que se halle el trabajador. Y cada empresa posee su propio estilo e imprimir su propio estilo a la dinámica del mercado.

Las crisis económicas, sociales, políticas y culturales impactan en el desempeño de la empresa y la confrontan con desafíos que amenazan su existencia pues desafían todo su saber y todas sus destrezas (cf. Guéhenno, 1995, pp. 111,71-72). Una crisis aguda puede llevar a una empresa a la banca rota y a su extinción. Aun cuando la empresa, luego de varios años de haber sido insertada en la realidad, haya ganado experiencia, los desafíos son una constante que permanece: es la apertura de la empresa hacia el futuro.

De la experiencia de haber afrontado desafíos tempranos, cuando apenas había atravesado su primera fase de formación, la empresa gana fortalezas que le permiten gestionar esa dimensión palpitante de los negocios, que marca el pulso de la empresa y al mismo tiempo le da insumos a la empresa para que, sobre la base de la investigación y el conocimiento objetivo de la realidad empresarial, pueda ensayar, experimental e innovar, mejorando la calidad de la empresa. La empresa aprende a ser empresa. La empresa que mira las crisis como una oportunidad para fortalecerse como empresa está mejor preparada que aquella otra que sucumbió ante la crisis y

\section{CONCLUSIONES}

no pudo recuperarse del impacto. Las lecciones puestas en limpio después de la crisis sacan en claro enseñanzas para el presente y el futuro.

Los estudios que nutren esta reflexión inciden en el carácter constructivo de la empresa como uno de sus factores aprovechables más fértiles para el fortalecimiento y la sostenibilidad de la empresa en el mundo de hoy, es decir de la empresa frente a sus desafíos presentes, vigentes y actuales. La reconsideración que la empresa hace de sus fines permite sacar a flote los fundamentos teóricos de la empresa moderna, así como extraer enseñanzas de la experiencia de sus momentos constitutivos, lo que permite superar el horizonte de los prejuicios tradicionales que desdibujan la imagen de la empresa como resultado de la cooperación y la colaboración entre las personas, la comunidad, la sociedad y el Estado.

Aunque la gran compañía capitalista trata a sus clientes de manera impersonal, abstracta y general sin atender a las particularidades específicas de cada uno de sus clientes, sumiéndolos en un anonimato práctico, lo que impacta en la configuración del espacio público como espacio reducido por lo "económico" y lo comercial, también se constata una tendencia creciente hacia la mayor personalización del servicio empresarial (cf. Sher, 2011). La empresa es cada vez más consciente de que la reducción del espacio público, es decir del espacio donde confluyen la eticidad y la juridicidad, socava la autonomía ciudadana y que a la larga la situación podría resultar perjudicial para los actores socioeconómicos. Sin embargo, la crisis del Estado - ocasionada en parte por su propio desempeño frente a la empresa, ocasionada en parte por la resistencia de la empresa frente al Estado- no anula ni suprime la relación entre la empresa y la eticidad. 


\section{REFERENCES}

Abalde, M. L. (2016). La publicidad personalizada, el futuro de la publicidad en televisión. Catalunya: Universitat Oberta de Catalunya. Trabajo Final de Grado, Grado de Comunicación.

Águila, L. del, sel. (2007). Ética, economía y empresa. En M. Giusti \& F. Tubino (Eds.), Debates en la ética contemporánea (pp. 211-256). Lima: PUCP.

Arellano, J. (2011). ¿Minería sin fronteras?: conflicto y desarrollo en regiones mineras del Perú. Lima: IEP, PUCP, \& UARM.

Arellano, J. (2013). Minería y conflicto en Perú: sembrar minerales, cosechar una avalancha de piedras. En A. Bebbington (Ed.), Industrias extractivas: conflicto social y dinámicas institucionales en la región andina (pp. 151184). Lima: IEP, Cepes, \& Grupo Propuesta Ciudadana.

Aristóteles. (2010). Ética a Nicómaco, trad. de Julio Pallí Bonet. Madrid: Gredos.

Beauchamp, T. (2000). Manipulative Advertising. En N. E. Bowie \& T. L. Beauchamp (Eds.), Ethical Theory and Business (pp. 476-485). New Jersey: Prentice Hall.

Bentham, J. (2012). The Principles of Morals and Legislation. In R. M. Baird \& S. E. Rosenbaum (Eds.), Nueva York: Prometheus Books.

Bohman, J. (1996). Public Deliberation. Pluralism, Complexity, and Democracy. Cambridge: MIT Press

Bourdieu, P. (1985). The Social Space and the Genesis of Groups. Theory and Society, 14(6), pp. 723-744.

Brescia, C. \& Quea, V. (2014). Minería e inversión social en turismo. Miradas desde la ética, el desarrollo y la sostenibilidad. En C. Del Mastro Puccio (Ed.), Desafíos éticos en los negocios (pp. 87-106). Lima: Universidad del Pacífico.

Calvo, C. (2014). Ética de las capacidades y desarrollo humano en las empresas. En C. Del Mastro Puccio (Ed.), Desafíos éticos en los negocios (pp. 11-35). Lima: Universidad del Pacífico.

Carson, T. (2001). Ethical Issues in Selling and Advertising. In N. E. Bowie (Ed.), The Blackwell Guide to Business Ethics (pp. 186-205). Oxford: Blackwell.

Cortina, A. (1994). Ética de la empresa. Claves para una nueva cultura empresarial. Madrid: Trotta.

Crocker, D. (2005). Sen and Deliberative Democracy. In A. Kaufman (Ed.), Capabilities Equality. Basic Issues and Problems (pp. 295-359). Nueva York: Routledge.

De Echave, J., \& Torres, V. (2005). Una estimación de los efectos de la minería en los índices de pobreza en Perú. Lima: CooperAcción.

Elster, J. (1997). The Market and the Forum: Three Varieties of Political Theory. In J. Bohman, \& W. Rehg (Eds.), Deliberative Democracy: Essays on Reason and Politics (pp. 4-33). Cambridge: MIT Press.
Forst, R. (2007). First Things First: Redistribution, Recognition and Justification. European Journal of Political Theory, 6(3), 291-304.

Fraser, N. (2007). Identity, Exclusion, and Critique. European Journal of Political Theory, 6(3), 305-338.

Goulet, D. (1995). Development Ethics: A Guide to Theory and Practice. Washington: Rowman \& Littlefield Publishers.

Groppa, O. (2005). El enfoque de las capacidades de Amartya Sen y Martha Nussbaum. Revista Erasmus, 1, 79-101.

Guéhenno, J.-M. (1995). El fin de la democracia. La crisis política y las nuevas reglas del juego. Barcelona: Paidós.

Habermas, J. (1992) Faktizität und Geltung. Frankfurt: Suhrkamp.

Habermas, J. (1996). Drei normative Modelle der Demokratie. In Die Einbeziehung des Anderen (SS. 277292). Frankfurt: Suhrkamp.

Habermas, J. (1999). La inclusión del otro. Estudios de teoría política. Barcelona, Madrid y México: Paidós.

Hegel, G.W.F. (1993). Fundamentos de la Filosofía del Derecho. Madrid: Libertarias y Prodhufi.

Honneth, A. (1992). Kampf um Anerkennung. Frankfurt: Suhrkamp

Honneth, A. (2003). Redistribution as Recognition. A Reply to Nancy Fraser. In N. Fraser, \& A. Honneth (Eds.), Redistribution or Recognition? London: Verso.

Honneth, A. (2010). Das Gewebe der Gerechtigkeit. In Das Ich im Wir (SS. 51-77). Berlin: Suhrkamp.

Knieper, R. (1991). Nationale Souveranitat. Frankfurt: Fischer.

Laden, A. (2007). Reasonable Deliberation, Constructive Power and the Struggle for Recognition. In B. Van den Brink and D. Owen, eds. Recognition and Power. Axel Honneth and the Tradition of Critical Social Theory, pp. 270-289. Cambridge: Cambridge University Press.

Luetge, C., ed. (2013). Handbook of the Philosophical Foundations of Business Ethics. Dordrecht, Heidelberg, New York \& London: Springer.

Mauss, M. (2009). Ensayo sobre el don. Forma y función del intercambio en las sociedades arcaicas. Buenos Aires: Katz.

Nussbaum, M. (2002). Virtudes no relativas: un enfoque aristotélico. En: Las mujeres y el desarrollo humano. El enfoque de las capacidades. Barcelona: Herder.

Pastor, C., Ángeles, E., Pérez, P., \& Palomino, M. (2012). Efecto de la minería sobre el empleo, el producto y la recaudación en el Perú. Lima: IPE. Fecha de consulta: 10/10/2013. <http://ipe.org.pe/documentos/efecto-de-la mineria-sobre-elempleo-el-producto-y-la-recaudacionen-el-peru-0> 
Phillips, M. (1994). The Inconclusive Ethical Case against Manipulative Advertising. Business and Professional Ethics Journal, 13(4), 31-64.

Rawls, J. (2001). Justice as Fairness. A Restatement. Cambridge: Harvard University Press.

Rendtorff, J. D. (2013). Basic Concepts of Philosophy of Management and of Corporations. In C. Luetge (Ed.), Handbook of the Philosophical Foundations of Business Ethics (pp. 1361-1385). Dordrecht, Heidelberg, New York, \& London: Springer.

Rendtorff, J. D., ed. (2017). Perspectives on Philosophy of Management and Business Ethics. Including a Special Section on Business and Human Rights. Cham, Switzerland: Springer.

Santuc, V. (1997). ¿Qué nos pasa? Ética y política hoy. Lima: CEDEP, \& UARM.

Scalzo, G. (2014). Génesis del pensamiento económico: dos visiones en pugna. Cauriensia, IX, pp. 341-374.

Scalzo, G. (2017). A Genealogy of a Gift. In J. D. Rendtorff (Ed.), Perspectives on Philosophy of Management and Business Ethics. Including a Special Section on Business and Human Rights (pp. 31-45). Cham, Switzerland: Springer.

Schmidt, E. (1995). Ética y negocios en América Latina. Lima: Universidad del Pacífico.

Sher, S. (2011). A Framework for Assessing Immorally Manipulative Marketing Tactics. Journal of Business Ethics, 102(1), 97-118.

Sobrevilla, D. (2006). Derecho y Moral. Ius et Praxis, (36-37), 229-239.

Sobrevilla, D. (2009). Veinticinco años de reflexión ética en el Perú (1985-2010). Solar, 5(5), 13-37.

Taylor, C. (1994). Ética de la autenticidad. Barcelona: Paidós.

Taylor, C. et al. (1993). El multiculturalismo y «la política del reconocimiento». México: FCE.

Villarán, A. (2014). Crítica al engaño y a la persuasión irracional en la publicidad comercial. En: C. Del Mastro Puccio (Ed.), Desafíos éticos en los negocios (pp. 11-35). Lima: Universidad del Pacífico.

Waide, J. (1987). The Making of Self and World in Advertising. Journal of Business Ethics, 6(2), 73-79.

Young, I. (2000). Inclusion and Democracy. Oxford: Oxford University Press.

Zamagni, S. (2004). L'ancoraggio etico dell responsabilitá sociale d'impresa. En: Actas del Convegno Internazionale Nuovi Orizzonti della Economia di Comunione, 20 de octubre. Castelgandolfo.

Zamagni, S. (2010). Fraternidad, don y reciprocidad en la "Caritas in Veritate". Revista de Cultura Económica, 27(75-76), 11-29. 